\title{
A Research in Key Generation Security and Accuracy of LIP Based Biometry
}

\author{
S.Pavithra, P.Muthukanna
}

\begin{abstract}
Authentication is the key factor of badging the right person for access which enhances the security system in wireless communication. In this paper, an efficient way of authenticating a person is achieved by selecting a novel biometric feature as -Lip\|. Series of image processing procedures are done to enhance the image features and also to fix the boundary values for corresponding pixels. A new filer termed as -Weighted Triangular Filter (WTF)\| is formulated to enhance the image features. Finally an 8 bit key is generated in lieu of biometric feature which is then used for encryption in wireless mode.
\end{abstract}

Keywords: Image concatenation, WTF filer, Key generation, MATLAB Simulation

\section{INTRODUCTION}

Biometrics

is related to body feature measurements are calculations. Biometric authentication is extracting the image features from the human physiological input, matching it with the database and allowing the grant for the right person to access the system. They are basically classified into physiological and behavioural characteristics. In recent scenario of the ever increasing population many people rely on networks for their routine lifestyle, the user data are more vital in real time environment in advent of this securing the data become more challenging task due to the ever increasing network traffic. Another most challenging task is that delivering the data to a right authenticated person at right time. In dynamic environment the intrusion of hackers are the watchdogs for secured data. Data securing and transmission to a right person is always challenging task. Few eminent physiological and behavioural features that can be used for authentication are listed below

\begin{tabular}{|c|c|}
\hline Psychological & Behavioural \\
\hline Face & Signature \\
\hline Finger Print & Voice \\
\hline IRIS & Key Stroke \\
\hline Hand Geometry & \\
\hline DNA & \\
\hline Retina & \\
\hline
\end{tabular}

In connection with the existing features, this paper proposes an authentication system which uses human physiological feature - LIP $\|$ as an identity. In addition an 8bit key is generated in lieu with the LIP image to enhance the security of the system.
Key aspects on selecting "LIP" as the Biometric Feature

1. The shape and colour will be persistent in all image processing procedures.

2. The acquisition of lip image is very much viable in dynamic environment

3. Boundary analysis and image pixel conversion can be done easily

These images can be captured by using local cameras.

Sequence of Workflow

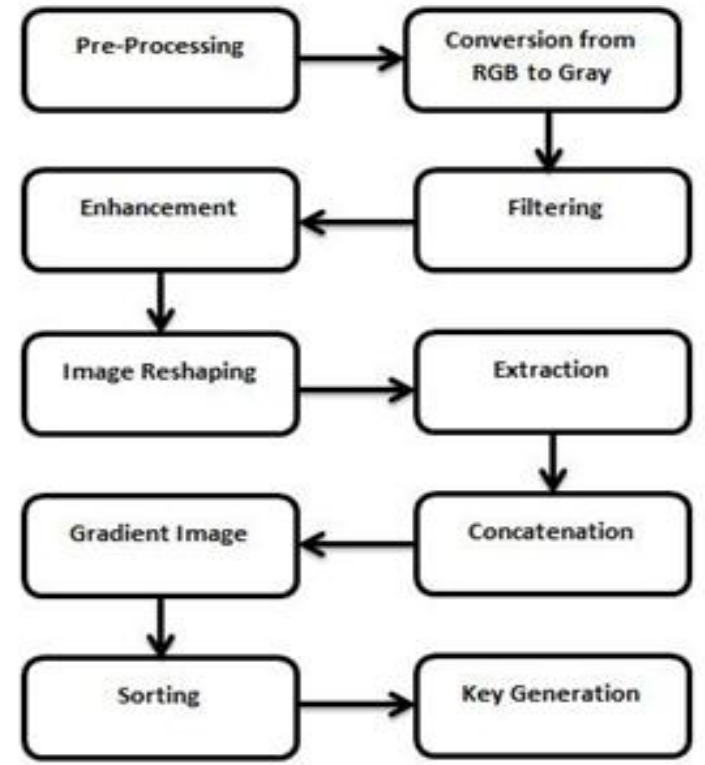

\section{FORMULATION OF KEY GENERATION}

\section{A. Pre-processing}

The image is fetched as JPEG format from the source as shown in Fig 2. RGB

Zero Matrix of empty space is created to fit the picture in the rows and columns by appending - $-[a, b] \|$

Variable _a $\mathrm{a}^{6}$ is appended to read the image.

\section{B. RGB to Gray Image Conversion}

After procuring the image, it is then converted into gray format as shown in Fig 3. Gray Image for detecting its outer lobe structure. Then histogram technique is applied to have to tonal distribution in the digital image by plotting the tonal value of each pixel which determines the pixel variation of the image. 


\section{A Research in Key Generation Security and Accuracy of LIP Based Biometry}

\section{Filtering}

In this procedure the image is fine-tuned by removing its noise elements. Noise is always an inseparable parameter of image and it ranges from Gaussian, Salt, Pepper, Speckle types based on the occurrence on the image. The noise from the dynamic image is removed by median filter. The essential features of median filter is that it preserves the edges of the image during the filtering process and it also run through the pixel entry by replacing the entries with the median of neighbouring entries.

In the paper, a novel method of filtering technique termed as -Weighted Triangular Filter (WTF)\| is used to fine tune the noise elements in the image. The key essential feature of triangular filter is that allotting the weight to each pixel which tends to have uniqueness over the original image. The triangle elements of the matrix image are considered for computation. When WTF filter is applied over an image the filtration ratio is better when compared with median and mean filters.

For evidence, initially the Gray image is exposed to -Median filter\| (Fig.4 Median Filter), -Ideal filter\| (Fig.5 Ideal Filter), -Mean filter\| (Fig.6 Mean Filter) and at last a novel filter of -Weighted Triangular Filter (WTF)\| (Fig.7 Weighted Triangular Filter) is introduced for a better efficacy. Finally as an concluding evidence a comparison graph shows that - Weighted Triangular

Filter (WTF) if prominent in image noise removal.(Fig.8 Comparison graph of filters)

\section{Enhancement}

The process of increasing the quality element of digitally stored image is called enhancement. In this process the image tonal level is adjusted by increasing/decreasing the contrast level of the image. In this paper, the WTF filter is used for image enhancement to mark a better fitted image with regularized tone for further processing.

E. Procedures involved in adjusting the intensity of the image

Intensity refers to the numerical value associated to the pixel. It the value is more, then the value of intensity is high and vice versa.

1. Analysing the lower and upper limit for the pixel and regularizing each pixel based on the tolerance value (fixing $0.5 \%$ )

2. Creating a matrix to accommodate 255 rows and 255 columns

3. Image to empty matrix fixation

4. Identifying the mean level of the image by dividing each pixel by 255

5. Adjusting each row and column pixel values by multiplying 255

The above procedures are performed for elemental division and multiplication for acquiring better enhanced image, shown in Fig.9 Enhanced Image.

\section{F. $\quad$ Featuring Extraction}

It sets the initial intend of measured data and builds derived values which are intended to be informative. Retrieving the originality of the image from the processed nature is called extraction. Feature extraction is to make the image to have a better pixel contrast with the initial set of measured data and delivering the content with informative supply that enhances the non-redundant nature of the pixel. The following sequence is followed to extract the image.

1. Cropping the image

2. Applying centroids to find the focal point of the image

3. Applying Concatenation - Using the command to fix the two appended images onto a single image.

Plot the markings points of the extracted image.

By using the MatLab code - region popsll the centroids of the image is formulated, this weighted centroid is appended to find the grey level of the image as shown in (Fig.10 Centroid Fixation prominent on centre Lip). Apart from this, the direction area of the lip image is also extracted to map the image vector direction, as shown in (Fig.11. Direction Area Features of Lip), which will acquire the value of extracted image and also to illustrate the area in which the traces of binary key generation can be done, as shown in (Fig.12 Area Features of Lip)

\section{G. Concatenation}

After fixing the image centroids and extracting the direction area, a separate concatenation process is done to append the two images onto a single image to make a clear focus on the centre part of the Lip. This midpoint focuses the system to formulate a binary key corresponding to the pixel value of the image. This foal point determines the authentic nature of the person's lip image.

\section{H. Gradient Extraction}

Image gradient is the gradual change in the intensity level of the image which has a gradual blend of colour with an even fading from low to high values, like changing from white to black regions. This is also called as colour progression. In this paper, the gradient extraction is done to enrich the baseline of the concatenated image which makes the best fit for the binary key generation.

\section{Sorting and Key generation}

The image after gradient extraction is then sorted by calculating the number of match points between image pixels to find the nearest pixel of the certain one. After calculating the matching points one by one, the accurate pixel variety is obtained for binary key formulation.

\section{Binary Key Generation}

1. The gradient level co-occurrence matrix of $(2 \mathrm{D}$ Image) is calculated

2. Gradient level co-occurrence properties of cooccurrence matrix is also calculated

3. Summing-up of gradient level co-occurrence matrix and gradient level co-occurrence properties is done by rounding all the property with property matrix

4. Conversion of property matrix to binary values is performed

5. Maximum and Minimum values of the binary values are fixed by appending the required number of bits to 
the left of the binary values

6. Comparing all the binary bits position, to check the data relevance property (either 1 or 0 )

7. Fixing-up the data relevance in an array and finding out the matching position in that array

8. Finding the sum of the data relevance and matching position and to find the average

9. Rounding all the average value and limiting it to a binary level to 8-bit.

10. Fixing a string structure over the 8-bit binary key to have a sequential representation

The formulated binary key is shown in (Fig.13. Binary Key of 8bit length) which is of 8-bit in length, this key depicts the encrypted lip image with an elevated level of security possessed during network transfer.

\section{SIMULATION DEPICTION \& RESULTS}

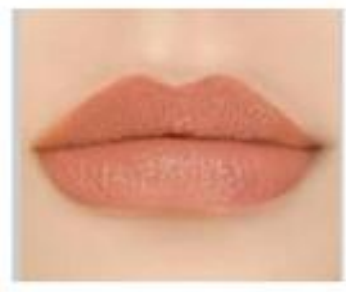

Fig. 2 - RGB

Image

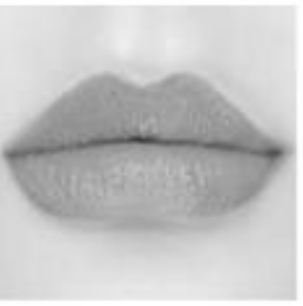

Fig.4 - Median

Filter

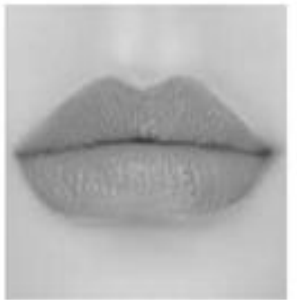

Fig.6 - Mean

Filter

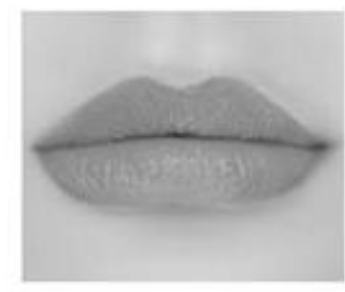

Fig.3 - Gray Image

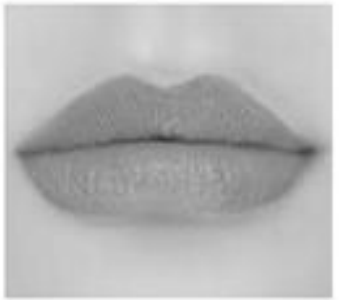

Fig.5 - Ideal

Filter

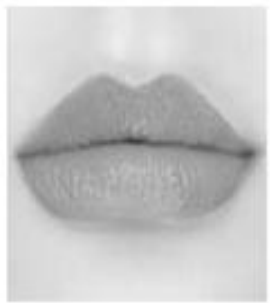

Fig.7 - Weighted

Triangular Filter

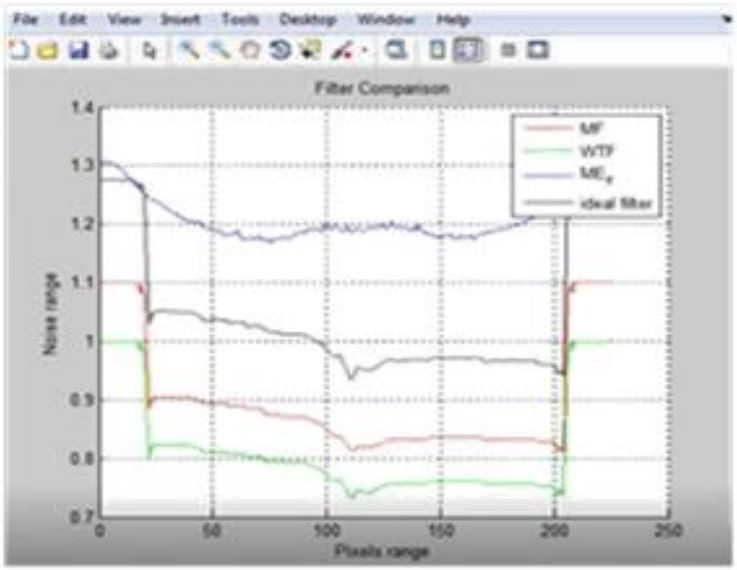

Figure 8 -Comparision of Mean, Median, Ideal \& Weighted Triangular Filter

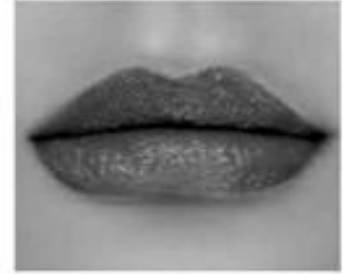

Fig 9: Enhanced image Filter

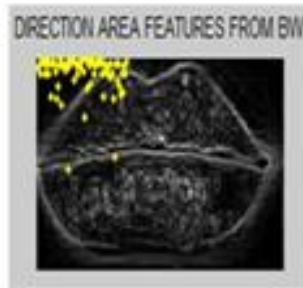

Fig 11:Direction Area Features of lip

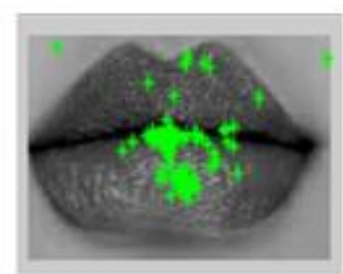

Fig 10: Centroid Fixation prominent on Centre lip

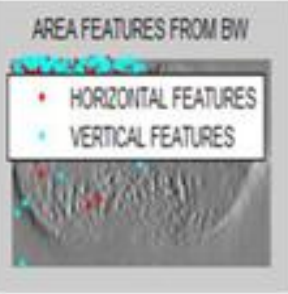

Fig 12: Area Features of Lip

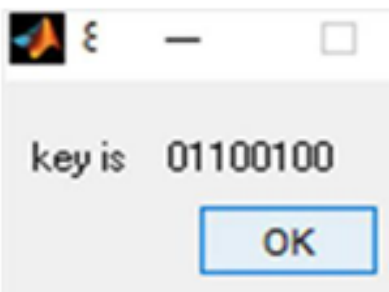

Fig.13 -Binary Key of 8bit in length 


\section{REFERENCES}

1. Abdeljebar Mansour; Mohamed Sadik; Essaï Sabir; MostafaJebbar-AMBAS:An Autonomous Multimodal Biometric Authentication System 2017. 13th International Wireless Communications and Mobile Computing Conference (IWCMC)-Year: 2017-Pages: 2098 - 2104-IEEE Conference Publications

2. Burge M., Burger W., -Ear Biometrics, Biometricsll: personal identification in networked society, in: A.K. Jain, R. Bolle, S. Pankanti (Eds.), 273-286 (2012).

3. Devesh Narayan, Sipi Dubey -A Survey Paper on human identification using ear biometricsl ,International Journal of Innovative Science and Modern Engineering (IJISME) ISSN: 2319-6386, Volume-2 Issue-10, September 2014.

4. Iannerelli A., -Ear Identification- Forensic Identification Seriesll, Paramount Publishing Company, Fremount, California (2012).

5. Raul Sanchez-Reillo; Helga C. Quiros-Sandoval; Ines Goicoechea-Telleria;-Improving Presentation Attack Detection in Dynamic Handwritten Signature Biometrics-Year: 2017, Volume: PP, Issue: 99

6. Manjari Benhar Peethala; Sujata Kulkarni-Integrating Biometric Cryptosystem with steganography for authentication-2016 IEEE International WIE Conference on Electrical and Computer Engineering (WIECONECE) Year: 2016 Pages: 28 - 31 IEEE Conference Publications

7. Sarika B. Solanke ; Ratnadeep R Deshmukh-Biometrics - Iris Recognition System\| A study of promising approaches for secured authentication-IEEE Xplore- 2016

8. Ziga Emersic, Vitomir Struc, Peter Peer, Ear Recognition: More than a Survey, Elsevier (2017). 